\title{
The role of feedback in the production of skilled finger sequences
}

\author{
Nicola J. Popp ${ }^{1}$, Carlos R. Hernandez-Castillo², Paul L. Gribble ${ }^{1,3,4,5}$ \& Jörn \\ Diedrichsen ${ }^{1,6,7^{*}}$
}

\begin{abstract}
${ }^{1}$ The Brain and Mind Institute, University of Western Ontario, Canada,
${ }^{2}$ Faculty of Computer Science, Dalhousie University, Canada,

${ }^{3}$ Department of Psychology, University of Western Ontario, Canada

${ }^{4}$ Department of Physiology \& Pharmacology, University of Western Ontario, Canada,

${ }^{5}$ Haskins Laboratories, USA,

${ }^{6}$ Department of Statistical and Actuarial Sciences, University of Western Ontario, Canada,

${ }^{7}$ Department of Computer Science, University of Western Ontario, Canada
\end{abstract}

\section{Acknowledgments}

This work is supported by a Canada First Research Excellence Fund (BrainsCAN) to Western University, a Natural Sciences and Engineering Council of Canada (NSERC) Discovery Grant (RGPIN-2016-04890) to J.D., a NSERC Grant (RGPIN 238338), a Canadian Institutes of Health Research Grant (PJT-153447), and a National Institute of Child Health and Human Development Grant (R01 HD075740) to P.L.G.

\section{Correspondence address}

Jörn Diedrichsen, The Brain and Mind Institute, Western University, London, Canada.

\section{idiedric@uwo.ca}

\section{Author contributions}

N.J.P., J.D. \& P.L.G. designed the experiment. N.J.P, C. R. H-C. and J.D. programmed the experiment. N.J.P collected the data and analyzed the data. N.J.P., J.D., and P.L.G. wrote the manuscript.

\section{Conflict of interest}

The authors declare no conflict of interest. 


\section{Abstract}

2 Actions involving fine control of the hand, for example grasping an object, rely heavily on

3 sensory information from the fingertips. While the integration of feedback during execution

4 of individual movements is well understood, less is known about the use of sensory

5 feedback in the control of skilled movement sequences. To address this gap, we trained

6 participants to produce sequences of finger movements on a keyboard-like device over a

7 four-day training period. Participants received haptic, visual, and auditory feedback

8 indicating the occurrence of each finger press. We then either transiently delayed or

9 advanced the feedback for a single press by a small amount of time (30 or $60 \mathrm{~ms})$. We

10 observed that participants rapidly adjusted their ongoing finger press by either

11 accelerating or prolonging the ongoing press, in accordance with the direction of the

12 perturbation. Furthermore, we could show that this rapid behavioural modulation was

13 driven by haptic feedback. While these feedback-driven adjustments reduced in size with

14 practice, they were still clearly present at the end of training. In contrast to the

15 directionally-specific effect we observed on the perturbed press, a feedback perturbation

16 resulted in a delayed onset of the subsequent presses irrespective of perturbation

17 direction or feedback modality. This observation is consistent with a hierarchical

18 organization of skilled movement sequences, with different levels reacting distinctly to sensory perturbations. 

and feel a sudden slip, we can swiftly adjust our grip force to avoid the cup slipping from

24 our hand. This correction can occur in less than $100 \mathrm{~ms}$ (Cole and Abbs 1988;

25 Hernandez-Castillo et al. 2020; Johansson et al. 1992). Feedback from other senses

26 such as vision (Day and Lyon 2000; Veerman et al. 2008) and audition (Burnett et al.

27 1998; Howell 2004) is also used for the control of an ongoing movements, albeit at

28 slightly slower speeds (at 90-260 ms and 100-200 ms respectively). Based on the

29 importance of sensory feedback, researchers have proposed that continuous feedback integration is essential for accurate movement execution (Adams 1971). While much is known about the rapid sensory feedback integration during the

32 execution of individual movements (for reviews see Cluff, Crevecoeur, \& Scott, 2015;

33 Scott, 2012; Shadmehr, Smith, \& Krakauer, 2010), less is known about the integration

34 of sensory feedback during the execution of sequences of finger movements. Previous

35 studies investigating this topic have primarily focused on tasks in which participants 36 were asked to synchronize their movements with an external pacing tone (Aschersleben

37 2002; Gates et al. 1974; Kulpa and Pfordresher 2013; Pfordresher and Benitez 2007;

38 Repp 2000; van der Steen et al. 2014). Studies investigating the role of sensory

39 feedback in tasks in which participants execute finger movements as fast as possible,

40 however, are scarce (Jay and Hubbold 2005; Long 1975). Moreover, the majority of

41 studies investigating this topic have focused on perturbing the slower visual or auditory

42 feedback channels. Hence, these studies were unable to examine the full range of rapid

43 feedback adjustments that are possible during a finger press. 
Here we probed the use of sensory feedback during the execution of fast finger movement sequences. We manipulated haptic, visual, and auditory feedback on a few selected presses within a sequence, in a way that was not consciously perceivable by

47 the vast majority of participants. Participants were trained on sequences of finger movements on an isometric keyboard throughout a four-day training period. On each press, upon reaching a given force threshold, participants were given a small haptic stimulus, similar to the feedback devices embedded in modern computer trackpads or

51 smartphones. Concurrently, auditory and visual feedback indicated the successful

52 pressing of the key. We then either delayed or advanced feedback on a single press

53 within a sequence to probe how this sensory feedback is used in control. During the

54 delayed feedback perturbation participants were not required to wait for the feedback to

55 perform the subsequent presses - thus, by design, they could perform the task without

56 considering feedback. However, we found an immediate, directionally-specific reaction

57 to the feedback perturbation, providing strong evidence for the reliance of fast finger

58 sequences on feedback.

The way participants react to a small feedback perturbation also provides a probe into how skilled motor sequences are organized. Models of sequence

61 performance usually fall on a continuum along two extremes (Diedrichsen and

62 Kornysheva 2015). On one side, sequences are controlled as a single unit or motor

63 program (Keele 1968) that specifies the detailed muscle commands necessary to

64 produce the sequence (Fig. 1a). On the other end is the idea that movement sequences

65 are controlled hierarchically (Rosenbaum et al. 1983), in which one more abstract layer 
66 represents the sequence to be executed and a lower-level layer generates the detailed

67 muscle commands for each finger press (Fig. 1b).

While both models would predict a modulation of the press that is perturbed, they

69 differ in how subsequent presses would be affected. In the single motor program model,

70 an acceleration or delay of a single movement element will shift the subsequent presses

71 accordingly. In contrast, in the hierarchical model, the influence of a local sensory

72 perturbation on a single finger could differ from the influence on subsequent presses.

73 How exactly subsequent presses are influenced depends on how feedback is

74 communicated from the lower-level finger controllers to the higher-level sequence

75 controller (Kiebel et al. 2009), and how the sequence controller uses the feedback. By

76 comparing the influence of a sensory feedback perturbation across finger movements of

77 a sequence, we are able to gain novel insights into how sensory feedback is used in this

78 organization. 
a

Single motor program

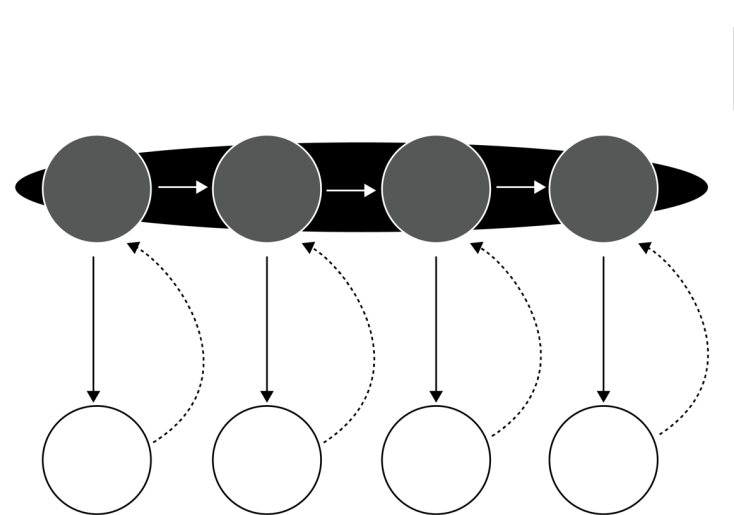

b Hierarchical controller

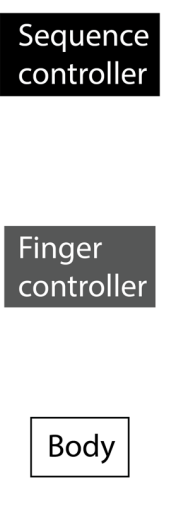

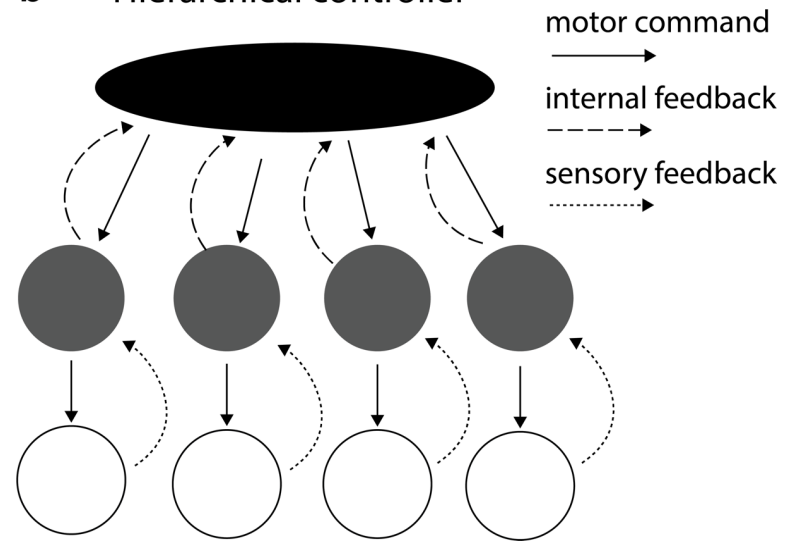

Figure 1. Two hypothetical representations of movement sequences. (a) A single motor program represents the movement sequence as an integrated unit. The completion of one finger controller automatically triggers the next finger controller. (b) A hierarchical controller represents the movement sequence across multiple layers that interact to produce the sequence of movements. The finger controllers represent the specific muscle commands for each of the fingers and are responsible for finger press execution. The sequence controller commands the finger controllers to initiate movements. In this particular model, the finger controllers provide internal feedback to the sequence controller when the finger press is completed. However, the next press may be initiated at a different time from the occurrence of the internal feedback.

\section{Methods}

80

81

82

83

84

85

87

86 board of the University of Western Ontario and all participants gave their signed consent

\section{Participants}

Twenty-six participants were recruited for this study (11 males; ages 18 to 44;

mean age 25.5 [ \pm 7.25$])$. All participants were right-handed (self-declared) and

completed informed consent. On average participants had received $6.44( \pm 7.25)$ years

of musical training based on their longest played instrument, with $57 \%$ having at least

one year of piano playing experience. The study protocol was approved by the ethics

7 before starting the study. 
$\underline{\text { Apparatus }}$

To test participants, we used a custom-built five-finger keyboard (Fig. 2a). The

90 keys were not depressible but a force transducer (FSG-15N1A, Sensing and Control,

91 Honeywell) was mounted underneath each key measuring isometric force production

92 with a repeatability of $<0.02 \mathrm{~N}$ and a dynamic range of $16 \mathrm{~N}$ (Wiestler et al. 2014;

93 Wiestler and Diedrichsen 2013; Yokoi et al. 2017). The digital sampling rate of the

94 measured force was $200 \mathrm{~Hz}$. Additionally, each key was equipped with a linear resonant

95 actuator (LRA, LVM061930B-L20, Jinlong Machinery \& electronics Inc.) that provided

96 haptic feedback during the experiment. LRAs vibrate at a frequency between 200 and

$97250 \mathrm{~Hz}$. In our application, a haptic controller creates a specific waveform to elicit the

98 click sensation. The haptic stimulation was produced by a haptic motor controller

99 (DRV2605L, Adafruit Industries LLC) that produces a computer-controlled click/vibratory

100 sensation that feels similar to the sensation experienced from smartphone keys or

101 trackpads on laptops (see the DRV2605L dataset for more information regarding the

102 specific waveform).

103 
a

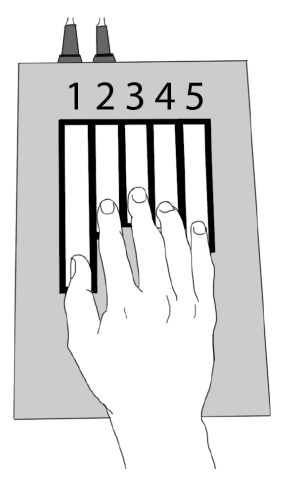

b

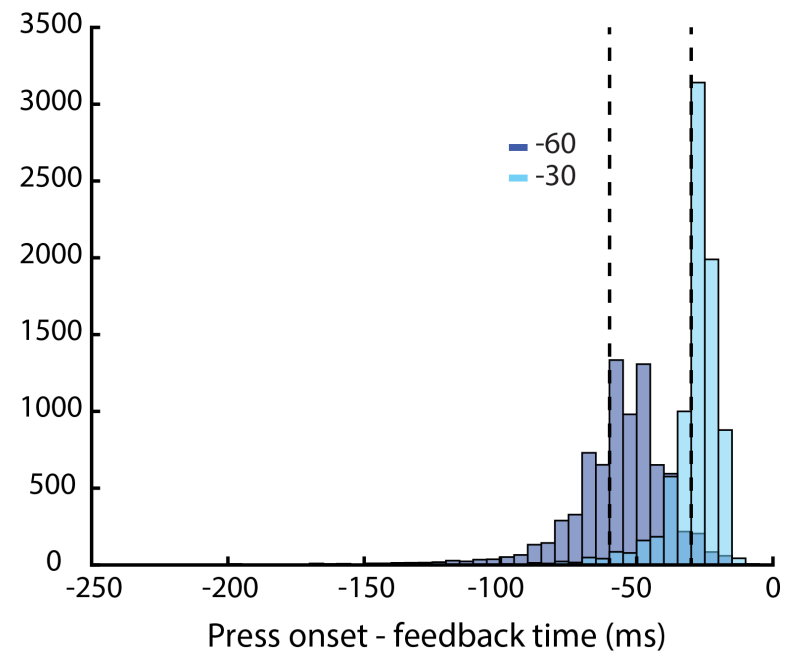

Figure 2. Apparatus and achieved time advancements of feedback. (a) Isometric keyboard-like device. Each key was associated with a number (these numbers were not shown to the participants but verbally explained). (b) Histogram of the time intervals between feedback presentation and the actual press onset for the two advancement conditions. Vertical doted lines indicate $-30 \mathrm{~ms}$ and $-60 \mathrm{~ms}$.

Discrete sequence production task

Participants performed a discrete sequence production task (DSP), executing sequences of 11 keypresses as fast and as accurately as possible. Participants were instructed to move as fast as possible while maintaining an error rate of under $15 \%$ for each block of trials. Each finger was associated with a number (thumb $=1$, index $=2$, middle $=3$, ring $=4 \&$ little $=5$ ). Each trial began with the presentation of a sequence of numbers on a computer screen (white font). A trial was deemed completed after 11 finger presses were executed. The numbers stayed on the screen throughout execution. Participants performed three sequences in total that were randomly presented to

113 the participant. None of the sequences had directly repeating numbers (i.e., 33 or 44).

114 The same three sequences were used for all participants; however, the presentation 
115 order was randomized across participants. Each block consisted of 39 trials and each sequence was presented 13 times during a block.

The force magnitude applied to each key by the participant was displayed as five

118 lines on an LCD monitor, where each line height indicated the amount of force applied

119 to the corresponding key. When the force on a key exceeded $1.5 \mathrm{~N}$, the keypress was

120 registered and the feedback was triggered. Some co-articulation between fingers

121 emerged as the next key could be pressed before the previous key was released.

When participants pressed the correct key, the visual cue on the screen turned

123 green, a short pleasant auditory sound could be heard (each key was assigned a

124 specific tone that was different from the rest) and a small click could be felt on the

125 finger. If, however, an incorrect key was pressed, the visual cue changed to red, a

126 lower-pitch sound could be heard (same across keys), and a click (same for accurate

127 and incorrect press) could be felt.

For each completed trial participants received points based on their performance.

129 If the participant pressed all keys correctly and their median movement speed (MT - the

130 time between the first press and last release) was within $95 \%$ to $110 \%$ of the current

131 speed threshold (MT threshold) they received one point. If they correctly executed the

132 sequence and their median movement speed was faster than $95 \%$ of the current MT

133 threshold they received three points. If they pressed one or multiple keys incorrectly or

134 their median speed was slower than $110 \%$ of their MT threshold they received zero 135 points. At the end of a block, we provided participants with feedback regarding their

136 error rate, median speed (MT), points obtained for the current block, and total points

137 obtained across the session. To motivate participants to improve their performance 
138 throughout the sessions, we first set the MT threshold at $10 \mathrm{~s}$ at the beginning of each session and then adjusted it by lowering it to the median MT of a given block if the participant had a lower median MT compared to the current MT threshold and if their error rate was below $15 \%$.

\section{2}

\section{Feedback manipulation}

The first three blocks in each session were completely unperturbed, meaning no

144 feedback perturbation was presented. In each of the following blocks, we perturbed 24

145 trials out of the 39 trials. Participants completed a total of 74 blocks over the four days

146 of training. On these perturbation trials, we either advanced or delayed the haptic,

147 visual, and auditory feedback by 30 or $60 \mathrm{~ms}$ on one of the 11 key presses. To generalize our findings across fingers and press location within the sequence, we chose

149 two fixed positions within each sequence where feedback perturbations were given.

150 This also reduced the potential predictability of the perturbation location in each sequence. In sequence 1, we gave the feedback perturbation either at position 6 (finger 5) or 9 (finger 4 ), in sequence 2 at positions 4 (finger 2) or 7 (finger 1), and in sequence 3 at positions 5 (finger 4 ) or 8 (finger 3 ). In total, we presented the perturbation at six

154 different sequence positions across all sequences.

156 feedback had to be given to occur either 30 or 60 ms before press onset (the time at

157 which the force on the key exceeded 1.5 N). This prediction was updated in real-time

158 every 2 ms during trial execution. This prediction was based on three factors: the

159 current force, the current force change (numerical derivative based on three time 
161 model for each subject, sequence position, and delay condition (-30 ms or -60 ms)

162 using a logistic regression. This was done twice in each session. The first time we fit the

163 model on the data from the first three blocks, using the unperturbed trials as training

164 data. To account for speed changes during the session, we repeated the estimation in

165 the middle of the session based on the unperturbed trials of all previous blocks

166 (excluding the three initial blocks and at least six blocks of trials). The predicted

167 outcome variable was zero if it was too early to present feedback and one if it was too

168 late. Feedback was provided once the predicted probability exceeded 0.5 .

169 This approach led to an average time advancement of $29.3 \mathrm{~ms}$ (SD: $11.4 \mathrm{~ms}$ ) for

170 the $-30 \mathrm{~ms}$ advancement condition and an average of $57.9 \mathrm{~ms}$ (SD: $23.3 \mathrm{~ms}$ )

171 advancement for the $-60 \mathrm{~ms}$ condition (see Fig. 2b).

172 On the advanced trials, participants could press the next key as soon as the

173 feedback was presented on the current press, meaning they were allowed to press the

174 next key before reaching the press threshold for the perturbed press. This led to an

175 average of $2.36 \%$ (SD: $1.55 \%$ ) of the advanced trials not reaching the press threshold.

176 We excluded these trials from our analyses. Our analyses centred on calculating time

177 intervals between specific press landmarks and the press onset of the perturbed press.

178 In these trials the press onset was absent and thus we were unable to perform the

179 same analyses.

180 In the delay conditions feedback was withheld upon reaching the press threshold,

181 and instead presented 30 or $60 \mathrm{~ms}$ after press onset. However, in the delay conditions

182 participants were not required to wait for the feedback to be presented before moving

183 on to press the next press. This was important as participants did not have to take the 
184 feedback perturbation into account and could potentially perform the sequences just as

185 fast as when no perturbation was present.

\section{Experimental Procedure} depending on how fast the participant was able to complete the required blocks of trials. Participants completed one session per day and the four sessions were scheduled over a time span of approximately two weeks. We encouraged participants to take breaks

191 between blocks as necessary and offered a longer break in the middle of the experimental session. The participants were told that the goal was to perform the

193 sequences as accurately and fast as possible. At the end of the four sessions, we asked participants several questions about their experience that became more and more specific (see Appendix 1). This questionnaire was used to determine whether participants were conscious of the experimental manipulation. Only two participants expressed clear conscious knowledge of the experimental manipulation, while the rest

198 of the participants did not notice the manipulation. The performance of these two 199 participants was similar to the performance of the other participants and therefore were 200 not excluded from the analyses. Overall, the majority of participants were not 201 consciously aware of our experimental manipulation, and hence we believe that they did 202 not change their behaviour consciously.

$\underline{\text { Statistical Analysis }}$

For each trial, we calculated the overall movement speed (movement time/MT) 205 between the onset of the first press (first time it reached the press threshold) and the 
206 release of the last press (force fell below $1 \mathrm{~N}$ ). Additionally, we found five landmarks

207 (Fig. 3a) for each press: early onset ( $E O$ - when force first was great or equal to $0.75 \mathrm{~N}$ ),

208 onset ( $O$ - when force first was equal or exceeded $1.5 \mathrm{~N})$, peak $(P$ - time at highest force

209 - between onset and late release), release ( $R$ - when the force first fell under $1.5 \mathrm{~N}$ after

210 peak), and late release ( $L R$ - when force first fell under $0.75 \mathrm{~N}$ after onset). All analyses

211 were done relative to the onset of the perturbed press (or for unperturbed trials, the

212 matching unperturbed press in the same sequence). We analyzed the relative timing of

213 the landmarks on the perturbed press $(+0)$, and the two presses after the perturbed

214 press $(+1 \&+2)$.

215 All analyses were performed using custom-written code in MATLAB (The

216 MathWorks) and the dataframe toolbox (github.com/jdiedrichsen/dataframe). We

217 excluded any error trials from our analyses, as well as trials in which the press was

218 delayed by more than 100 ms after the advanced feedback was given, as we believe

219 that this could either suggest conscious awareness or an incorrect estimation from our

220 algorithm that predicts when feedback should be given. We analyzed the data using

221 paired one and two-sample t-tests that were based on clear a priori predictions and we

222 chose a probability threshold of $p<0.05$ for the rejection of the null hypothesis.

223 To estimate how quickly participants reacted to the delayed feedback by

224 adjusting the perturbed press, we conducted a change point analysis. We first

225 calculated the difference between the average force curves for the delayed trials

$226(+30 \mathrm{~ms}$ or $+60 \mathrm{~ms})$ and unperturbed trials from $20 \mathrm{~ms}$ before press onset and $240 \mathrm{~ms}$

227 after onset. Using the data before the occurrence of the peak difference between the

228 two curves, we estimated the time point when the difference started to emerge. We 
229 modelled the difference as a piece-wise linear function with a change point of $b_{0}$

230 between the two segments.

231

$$
\hat{y}(t)=\left\{\begin{aligned}
0, & t<b_{0} \\
\left(t-b_{0}\right) b_{1}, & t \geq b_{0}
\end{aligned}\right.
$$

232 where $\hat{y}(t)$ is the predicted force values for time $t, b_{0}$ is the chosen change point and $b_{1}$

233 is the slope of the function. Using the function fminsearch in MATLAB, we found the

234 values for $b_{0}$ and $b_{1}$ that minimized the sum of squares of the difference between

235 observed and fitted data.

237 the press following the perturbed press $(+1)$ would be delayed or accelerated (relative to

examined the difference in the effect of the perturbation at a singular point in time across the consecutive presses (i.e. a point in time where the force curves of the

241 presses overlap). We first chose a landmark at a time when the force curves of the two

242 presses overlapped. At the end of training, this overlap was clearly observed at the

243 onset $(1.5 \mathrm{~N})$ of the +1 press for the unperturbed trials, which we chose as our

244 reference landmark. On unperturbed trials, we then found the average force for the +0

245 press, which defined our matching landmark (i.e. that occurred at the same point in

246 time; see Fig. 3b). We then calculated the effect of the perturbation on these two

247 landmarks. The single motor program hypothesis predicts that both landmarks would be

248 delayed by the same amount of time (relative to an unperturbed press). In contrast, a

249 difference in delay (positive or negative) between the +1 press and the +0 press would

250 indicate that the effect of the perturbed feedback was not the same for the two presses. 
a

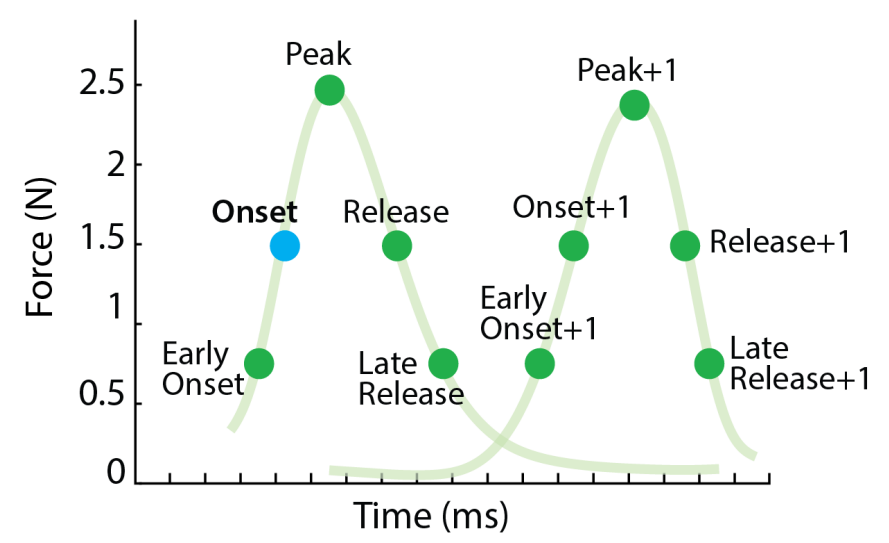

b

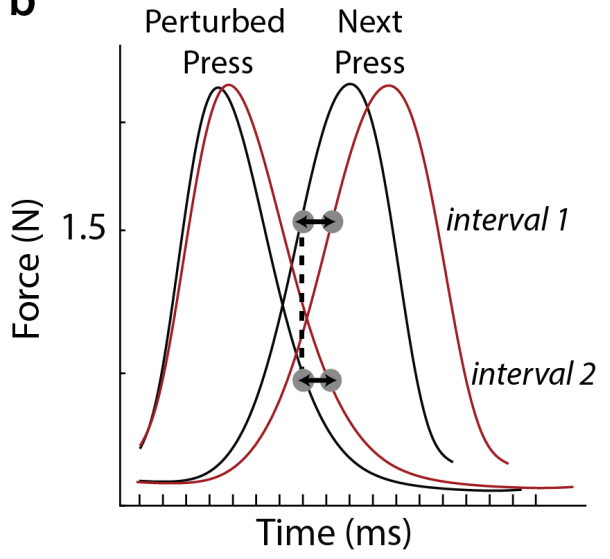

Figure 3. Calculation of feedback differences across presses and landmarks. (a) For our analyses we calculated time intervals between the onset of the perturbed press (blue onset dot in the figure) and different force landmarks (green dots) on the perturbed press as well as on subsequent presses (indicated with +1 ). We chose five specific force landmarks on each press: Early Onset $(>=0.75 \mathrm{~N})$, Onset $(>=1.5 \mathrm{~N}$ ), Peak (maximum $\mathrm{N}$ between onset and release of press), Release (first time $<1.5 \mathrm{~N}$ after onset), and Late Release (first time $<0.75$ after onset). (b) We choose a single time point (onset of next press) and compared how the perturbation affected this time point across presses. The black line indicates unperturbed trials and red lines represent perturbed trials.

\section{Control experiment}

254 feedback (auditory, haptic and visual) had differential effects on participants'

255 performance. We recruited 48 participants for this experiment. They were assigned to one of the three feedback groups (auditory, haptic or visual) at the beginning of training based on an algorithm that matched participants' speed, calculated as the time between the onset of the first press to the release of the last press (MT). This was done to ensure that the groups had similar average speeds at the start of the experiment. Participants only received one type of feedback throughout the study (how each

261 feedback was given was the same as described in the experimental design above).

262 When an incorrect finger press occurred, all groups saw the visual cue on the screen 
263 turn red to make it easier for them to know where they made the error in the sequence.

264 Participants practiced four different sequences (three were the same as in the main

265 experiment) for five days on the same keyboard-like device. Press threshold was $1 \mathrm{~N}$.

266 Because of the difference in press threshold we adjusted our landmark criteria for this

267 experiment: early onset ( $E O$ - when force first was great or equal to $0.6 \mathrm{~N})$, onset $(\mathrm{O}$ -

268 when force first was equal or exceeded $1 \mathrm{~N})$, peak $(P$ - time at highest force - between

269 onset and late release), release ( $R$ - when the force first fell under $1 \mathrm{~N}$ after peak), and

270 late release ( $L R$ - when force first fell under $0.6 \mathrm{~N}$ after onset). Feedback perturbations

271 were given on a single press within the sequence at two possible locations (similar to

272 the main experiment but the locations were not identical). In this experiment, we only

273 perturbed participants' feedback by delaying it by $80 \mathrm{~ms}$. The rest of the experimental

274 design was identical to the main experiment (point system, threshold change etc.). As in

275 the main experiment, most participants were unaware of the perturbation when asked

276 about it using a questionnaire at the end of the sessions.

\section{Results}

278 Feedback perturbations cause directionally specific behavioural adjustments to the

279 perturbed finger press.

280 To investigate how sensory feedback is used during the execution of fast finger

281 sequences, we used transient perturbations of the sensory feedback that indicated the

282 successful pressing of a key. The perturbation was only applied to a single press within

283 a sequence. Participants practiced three different sequences over four days. If sensory

284 feedback is used to control the near-isometric keypress, the delay and advancements of 285 feedback should prolong or shorten the ongoing press, respectively. 
The group average force traces (Fig. 4a) indicated that even though each finger press was completed within $\sim 300 \mathrm{~ms}$, participants indeed reacted to the feedback perturbation by extending or shortening the ongoing press. To quantify this effect, we calculated the time interval between the onset (first time $>=1.5 \mathrm{~N}$ is reached) and the peak (onset-peak) of the perturbed press (Fig. 4b onset-peak), as well as the interval between the onset and the release (first time $<1.5 \mathrm{~N}$ after onset; Fig. $4 \mathrm{~b}$ onset-release). On day 1 , both the $+30 \mathrm{~ms}\left(\mathrm{t}_{(25)}=11.189, \mathrm{p}=1.59 \mathrm{e}-11\right)$ and the $+60 \mathrm{~ms}$ delay condition $\left(t_{(25)}=4.969, p=2.02 e-05\right)$ resulted in a longer onset-peak intervals. Similar effects can also be seen on the interval between onset and release $\left(+30 m s: t_{(25)}=6.630, p=\right.$ 3.01e-07, +60 ms: $\left.\mathrm{t}_{(25)}=5.963, \mathrm{p}=1.58 \mathrm{e}-06\right)$. For the time advanced feedback conditions, the onset-release intervals on day 1 were shortened in response to perturbations (onset-release $-30 \mathrm{~ms}^{\mathrm{t}} \mathrm{t}_{(25)}=5.308, \mathrm{p}=8.42 \mathrm{e}-06$; $-60 \mathrm{~ms}: \mathrm{t}_{(25)}=4.291$, $\mathrm{t}=$ successful pressing of a key to finely control the duration of the force production.

Perturbation effects diminish but do not disappear with training practiced finger movements, we expect the feedback perturbation to still impact the duration of the press at the end of training. Indeed, this was what we found (Fig. 4a vs.

305 4c). Specifically, both delay conditions showed larger onset-peak intervals (+30 ms: $t_{(25)}$ $\left.306=5.963, p=1.17 \mathrm{e}-04 ;+60 m s: \mathrm{t}_{(25)}=6.420, \mathrm{p}=5.05 \mathrm{e}-07\right)$ and onset-release intervals $307\left(+30 m s: \mathrm{t}_{(25)}=6.143, \mathrm{p}=1.01 \mathrm{e}-06,+60\right.$ ms: $\left.\mathrm{t}_{(25)}=5.082, \mathrm{p}=1.51 \mathrm{e}-05\right)$ compared to 
309 intervals were observed for the advancement conditions (day 4 onset-release $-30 \mathrm{ms:}$ $\left.t_{(25)}=3.774, p=4.46 e-04,-60 m s: t_{(25)}=4.785, p=3.26 e-05\right)$. The finding of a clear

311 adjustment of the perturbed press at the end training suggests that even skilled

312 performance is controlled by sensory feedback.

313 While the overall effect was clearly present across all days, the effect caused by

314 the large perturbations reduced by $\sim 40 \%$. Specifically, the difference between perturbed 315 and unperturbed onset-release interval reduced from day 1 to day 4 for the $+60 \mathrm{~ms}(-$ $\left.31638 \%, \mathrm{t}_{(25)}=2.502, \mathrm{p}=0.019\right)$ and the $-60 \mathrm{~ms}$ condition $\left(-40 \% ; \mathrm{t}_{(25)}=-3.859, \mathrm{p}=7.106 \mathrm{e}-\right.$ 317 04). While the overall effect also reduced for the smaller perturbations, these changes 318 were not significant $\left(+30 \mathrm{~ms}:-29 \%, \mathrm{t}_{(25)}=1.848, \mathrm{p}=0.076 ;-30 \mathrm{~ms}:-35 \%, \mathrm{t}_{(25)}=-1.639\right.$, $319 p=0.113)$. This suggests that some transition from feedback to feed-forward control 320 took place in our task with practice. 
a

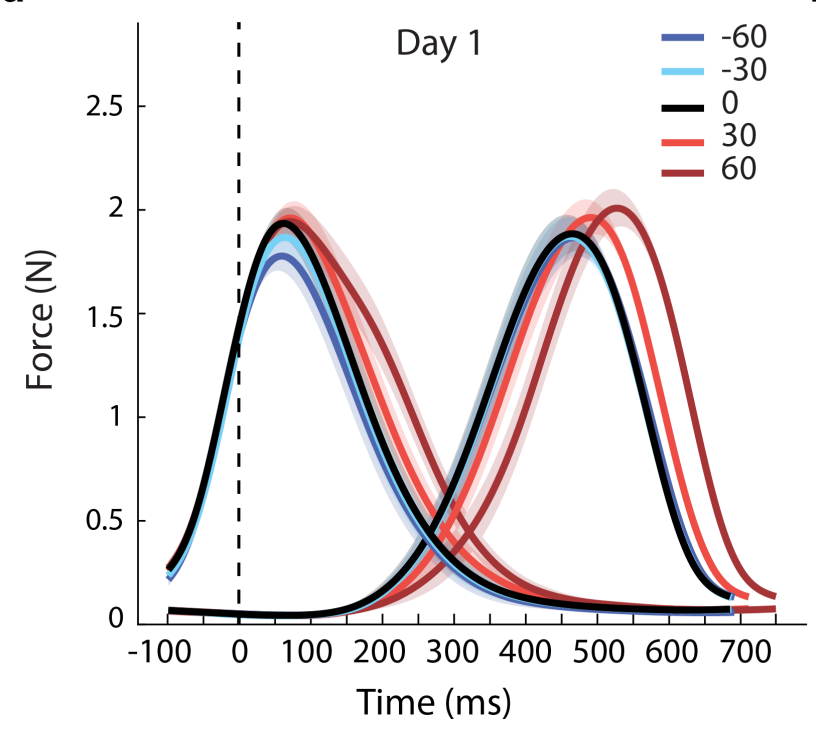

C

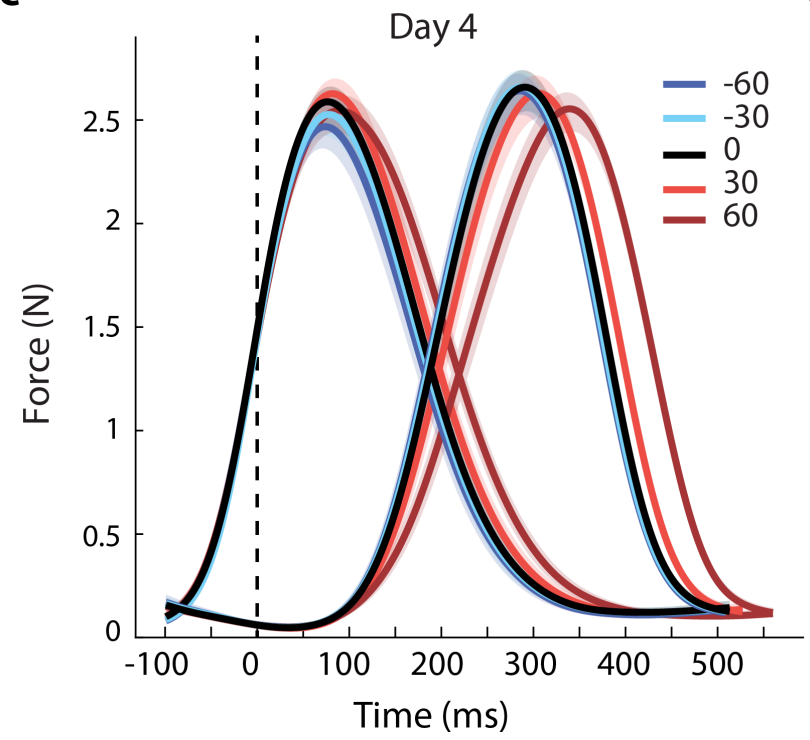

b

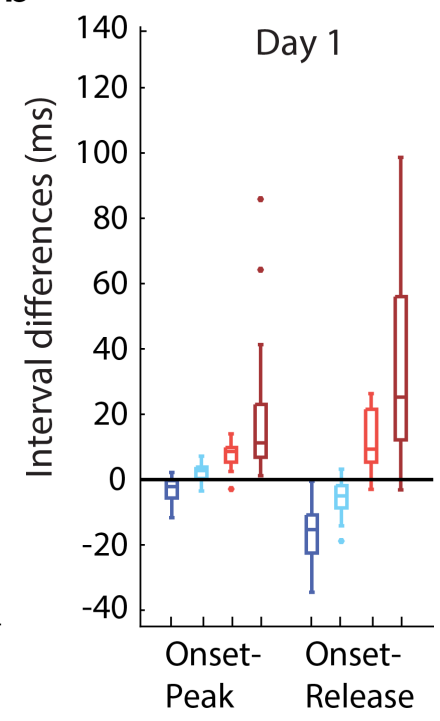

d

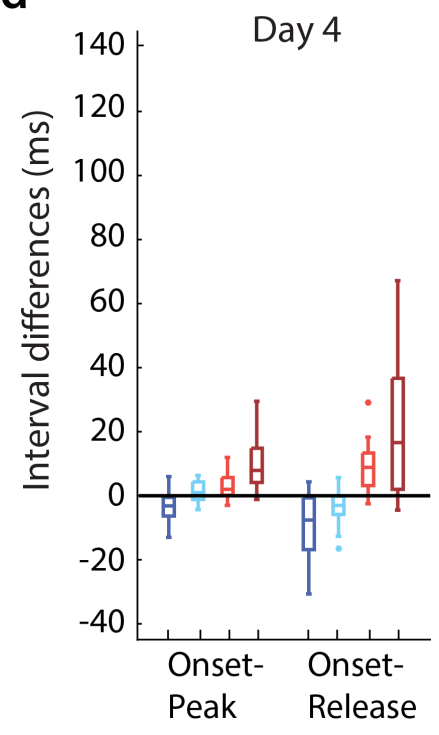

Figure 4. Effects of perturbation on perturbed press and subsequent press. (a) \& (b) Average force traces for day 1 and 4 and the following press interpolated and standardized to the average time of each condition. Dotted line indicates press onset, for which the sensory feedback was shifted in time. Error bars represent the standard error of the mean across subjects. (c) \& (d) Differences between the onset-to-peak and onset-to-release intervals of perturbed and unperturbed trials for day 1 and day 4. 


\section{Perturbations lead to reactions within $80 \mathrm{~ms}$}

324 press? To estimate this, we first calculated a difference curve between the average

325 force traces of the delayed perturbation conditions and the unperturbed condition for

326 each participant. We then used a change point analysis (see methods for details) to

327 estimate the time at which the difference curve was impacted by the feedback delay. On

328 day 1 in the $+60 \mathrm{~ms}$ delay condition, it took an average of $106.4 \mathrm{~ms}(95 \% \mathrm{Cl}[97.77$,

329 115.03]) after press onset for participants to show a divergence between the two force

330 traces. For the $+30 \mathrm{~ms}$ delay condition, the difference started at $77.3 \mathrm{~ms}(95 \% \mathrm{Cl}$

$331[64.65,90.04])$. For day 4 , our estimate of adjustment onset for the +60 ms condition

332 was $92.5 \mathrm{~ms}(95 \% \mathrm{Cl}[83.04,101.97])$, faster than day $1\left(\mathrm{t}_{(25)}=2.085, \mathrm{p}=0.047\right)$. The

333 estimate for the +30 ms condition was comparable to day 1 (mean: $67.5 ; 95 \% \mathrm{Cl}[46.32$,

334 88.72]; $\left.\mathrm{t}_{(25)}=0.738, \mathrm{p}=0.467\right)$. Overall, the adjustment of the ongoing press to the

335 delayed feedback was consistently very fast.

\section{Subsequent presses are delayed irrespective of perturbation direction}

So far, we have established that sensory feedback about the keypress is used to control the finger that produces the press, even during fast performance after extended training. Next, we investigated how the subsequent presses are impacted by the perturbation. This provides us with an opportunity to compare different models of how

341 skilled movement sequences are organized.

343 presses, we plotted the timing of five events (early onset, onset, peak, release, late 344 release, see Methods) for the perturbed and the two subsequent presses across the 
345 four sessions (Fig. 5). As the independent variable (i.e. x-axis) we plotted the groupaveraged time estimates of these landmarks for the non-perturbed trials relative to the onset of the perturbed press $(0 \mathrm{~ms})$. As the dependent variable (i.e. y-axis) we plotted the change in the average time interval relative to the unperturbed condition. Each press is indicated by a line that connects the five corresponding landmarks.

The feedback perturbations impacted not only the execution of the current press,

351 but also of subsequent presses. On the first day of training, both the $+30 \mathrm{~ms}$

352 perturbation $\left(\mathrm{t}_{(25)}=6.055, \mathrm{p}=2.51 \mathrm{e}-06\right)$ and the $+60 \mathrm{~ms}$ perturbation $\left(\mathrm{t}_{(25)}=9.078, \mathrm{p}=\right.$

2.177e-09) delayed the onset (interval onset-onset+1) of the next press relative to when no perturbation was present (i.e. red lines vs. grey line at zero). Moreover, the delay of feedback impacted even the onset of the press two positions after the perturbation $(+60$ $\left.m s: t_{(25)}=7.172, p=8.11 \mathrm{e}-08\right)$. In contrast, time advancements did not alter the timing of subsequent presses relative to the unperturbed trials (onset-onset+1: $-30 \mathrm{~ms}$ : $\mathrm{t}_{(25)}=-$ $\left.0.904 ; p=0.375 ;-60 m s: t_{(25)}=-1.488, p=0.149\right)$. This pattern of results provides new insights into how feedback is used in the control and representation of skilled movement sequences (as outlined in the introduction, Fig. 1). of one finger directly influences the control of the subsequent finger. This prediction becomes directly testable when there is considerable overlap, i.e. coarticulation, across

364 different finger presses. Such coarticulation was observed on days 3 and 4 (Fig. 5; where the onset of the second press roughly occurred at the same time as the release of the perturbed press). For such overlapping presses, the single motor program 
press and the onset of the next press in the sequence will be the same, even if the entire motor program is sped up or slowed down. In other words, the effect of the perturbation should be the same for simultaneous events on two overlapping presses.

371 To test this idea, we used the data from the last day of training. We compared the effect

372 of the perturbation on the onset of the next press (onest+1 Fig 5) with its effect on the

373 perturbed press at the same point in time (see Methods for detail). We found a

374 significantly longer delay for the subsequent press in comparison to the perturbed press

375 for the +60 condition $\left(t_{(25)}=2.522, p=0.018\right)$. This effect can be seen as an offset

376 between the end of the line for the perturbed press and the onset of the line of the

377 subsequent press in Figure 5 (day 4). A similar offset between presses was also

378 present between the second and third press after the perturbation $\left(t_{(25)}=3.429, p=\right.$

379 0.002). These additional delays across presses resulted in an overall slower execution

380 speed for the entire sequence $\left(\mathrm{MT}\right.$; day $\left.4:+60 \mathrm{~ms}: \mathrm{t}_{(25)}=5.828, \mathrm{p}=4.456 \mathrm{e}-06\right)$. These

381 findings provide clear evidence against the idea that the sequence is represented as a

382 single motor program after training. Rather it argues for a hierarchical organization (Fig.

$3831 \mathrm{~b})$, in which the effects on the subsequent finger presses can differ from the effect on

384 the perturbed finger.

385 The participants' reactions to the other perturbation conditions provide us with

386 more detailed insight into how feedback is considered in this hierarchical organization.

387 Similarly to what we have observed for the +60 ms delay condition, an offset between

388 the different presses was also observed for time-advancement of the feedback by -60

$389 \mathrm{~ms}$ (dark blue in Fig. 5), although this effect did not reach significance $\left(\mathrm{t}_{(25)}=2.043, \mathrm{p}=\right.$

390 0.052). Nevertheless, the offset was significant when comparing the second and third 
391 press after the perturbation $\left(\mathrm{t}_{(25)}=3.877, \mathrm{p}=6.799 \mathrm{e}-04\right)$. In the $-60 \mathrm{~ms}$ perturbation

392 condition, these additional offsets did not result in a significant slowdown of the overall

393 sequence speed (Day $\left.4: \mathrm{t}_{(25)}=-0.858, \mathrm{p}=0.399\right)$, suggesting that the additional delays

394 of subsequent presses were cancelled out by the speed-up on the perturbed press. In

395 contrast to the \pm 60 ms feedback perturbations, no clear offset was present for the

$396 \pm 30$ ms perturbation condition (Fig. 5 - light blue and light red). Indeed, the comparison

397 did not reach statistical significance for either time delay $\left(+30 \mathrm{~ms}: \mathrm{t}_{(25)}=0.882, \mathrm{p}=\right.$

$3980.193)$ or advancement perturbation $\left(-30\right.$ ms: $\left._{(25)}=0.589, p=0.281\right)$. In sum, for larger

399 but not for smaller perturbations participants delayed subsequent presses after the

400 occurrence of a perturbation, irrespective of whether the sensory feedback was

401 advanced or delayed.

402 Overall, our findings suggest a hierarchical organization in which sensory

403 feedback acts in two qualitatively different ways. First, the timing of the feedback

404 directionally either lengthens or shortens the perturbed key press. Second, the

405 occurrence of a perturbation also appears to act in a directionally non-specific manner

406 slowing down the execution of future presses. This effect was stronger for larger (60)

407 compared to smaller (30) perturbations but did not depend on the direction of the

408 temporal shift. 

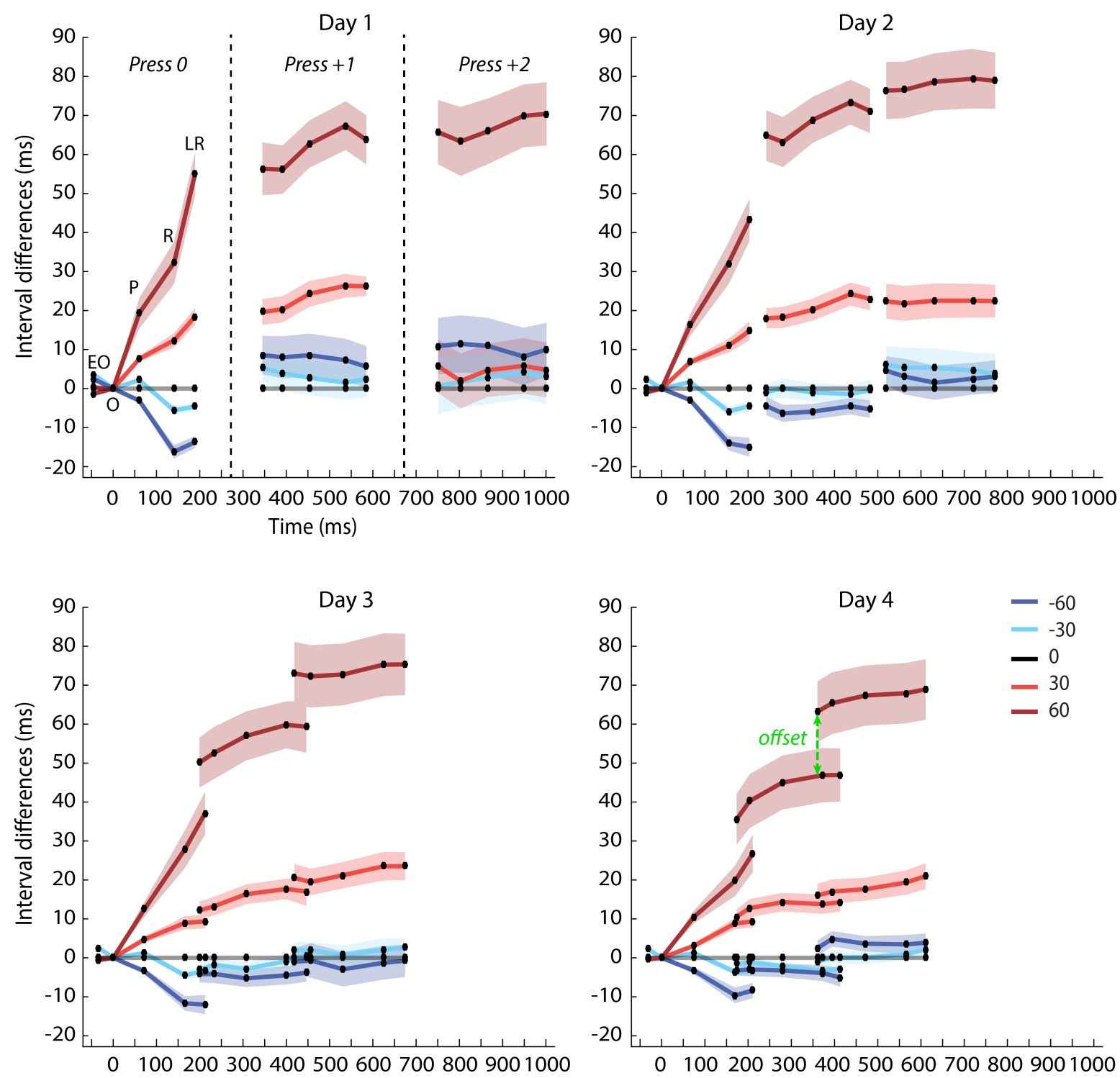

Figure 5. Effects of feedback perturbation on the perturbed press (press 0 ) and subsequent finger presses (Press +1, +2) across feedback conditions and training days. Five landmarks (EO: early onset, O: onset, P: peak, R: release, LR: later release) are plotted per press (see methods). The $x$-axis shows the average time of occurrence of the landmark on unperturbed trials relative to the onset of the first press. The $y$-axis shows the time interval differences between the perturbation conditions and the unperturbed condition on the particular landmarks. Landmarks belonging to a finger press are connected by a line. Anything above the 0 line indicates that the perturbation resulted in longer time intervals (i.e. slower) compared to when no perturbation was present, whereas everything below the line indicates shorter time intervals (i.e. speed-up). The different panels indicate the different training sessions (i.e. days). Day 4 shows how we tested the offset between presses, with an example of the $2^{\text {nd }}$ to $3^{\text {rd }}$ press for the $+60 \mathrm{~ms}$ condition. Error bars represent the standard error of the mean across participants. 


\section{$\underline{\text { Rapid behavioural adjustments are caused by haptic feedback }}$}

Finally, we investigated to what degree the effects observed in the main

412 experiment were due to the perturbation of haptic, visual, or auditory feedback. To test

413 this, we conducted a control experiment, in which a separate set of participants was

414 assigned to one of three experimental groups, with each group receiving only one of the

415 three types of feedback (auditory, visual or haptic). As in the main experiment, we

416 delayed the feedback on selected finger presses within the sequence. In this case, we

417 only chose a single perturbation condition (delay $+80 \mathrm{~ms}$ ) and participants practiced the

418 task for five days. Examining the effect of the delay on the perturbed press (see Fig. 6),

419 we found that only the haptic group demonstrated a significantly longer onset-peak

420 interval following the perturbation both in the beginning (Day $1: t_{(15)}=2.980, p=0.009$ )

421 and towards the end of training (Day $\left.4: \mathrm{t}_{(15)}=3.579, \mathrm{p}=0.003\right)$. Neither the visual (Day

$\left.4224: t_{(15)}=0.901, p=0.382\right)$ nor the auditory group (Day $\left.4: t_{(15)}=1.060, p=0.306\right)$

423 showed a significant effect of the feedback perturbation on the onset-peak interval.

424 These results clearly show that the rapid adjustments of the ongoing press were driven

425 by haptic feedback from the fingertip.

\section{Delay of subsequent presses arises from all three feedback modalities}

In contrast, the delay of subsequent presses was observed for all three feedback

428 modality groups. Consistent with the effect on the perturbed press, the delay of the

429 onset of the press following the perturbation (+1, averaged across days $2-5)$ was largest

430 in the haptic group $\left(69 \mathrm{~ms}, \mathrm{t}_{(15)}=6.890, \mathrm{p}=5.146 \mathrm{e}-06\right)$. However, both the auditory

431 group (35 ms, $\left.\mathrm{t}_{(15)}=4.888, \mathrm{p}=1.971 \mathrm{e}-04\right)$, as well as the visual group $\left(19 \mathrm{~ms}, \mathrm{t}_{(15)}=\right.$

$4324.828, p=2.214 \mathrm{e}-04$ ), showed a clear delay in the onset of the subsequent press, even 
433 though no such effect was observed on the perturbed press (Fig. 6). This result

434 suggests that the delay we observed on the subsequent presses in our main experiment

435 could be induced by the perturbations in each of the three feedback modalities.
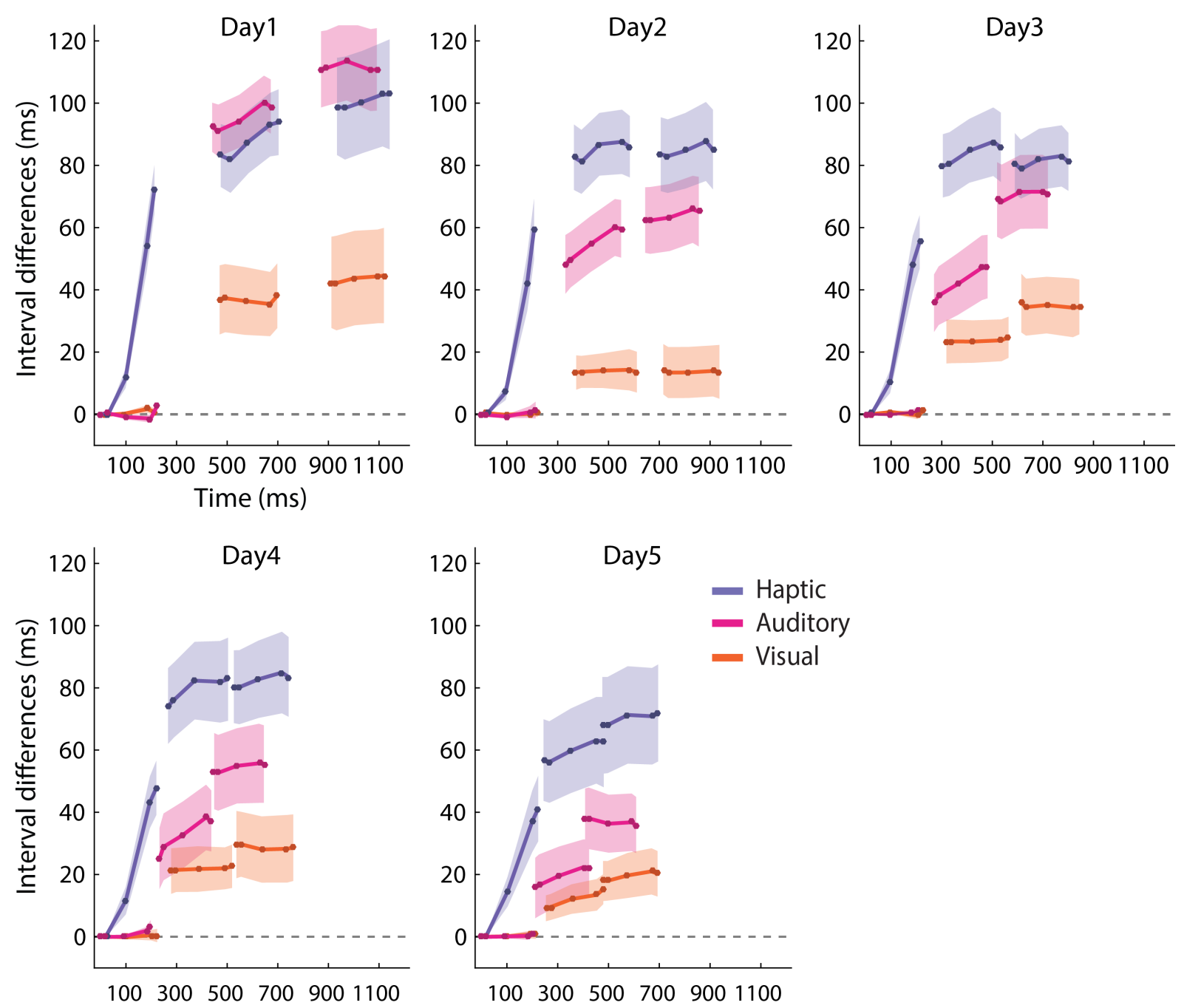

Figure 6. Effect of feedback perturbation for haptic, visual and auditory groups in control experiment across training days. As in Figure 5, five landmarks per press (connected by a line) are plotted. The control experiment only had $+80 \mathrm{~ms}$ perturbations, but each group received only one type of feedback. The different panels indicate the different training sessions (i.e. days). The error bars represent the standard error of the mean across participants for each group. 


\section{Discussion}

In this study, we used small transient feedback perturbations to probe how

sensory feedback is used in the control of fast finger movement sequences. Specifically,

441 we examined how sensory feedback modulates the execution of skilled finger

442 movements across four days of training, and how feedback differentially affects the

443 execution of the ongoing press and subsequent presses.

444 Sensory feedback rapidly modulates movement execution of the perturbed press

445 Throughout training, we found clear evidence of rapid behavioural adjustments of

446 the finger press that received the perturbation. This result illustrates the continuous

447 integration of sensory feedback when controlling skilled finger movements. Participants

448 adjusted their ongoing behaviour even though our task was designed so that it could be

449 accomplished without considering the feedback. The keypresses were isometric and

450 participants simply needed to exceed a specific force threshold. Nonetheless,

451 participants adjusted their behaviour based on the perturbation.

452 Furthermore, we found that the effects of the perturbation were directionally

453 specific: The delay in sensory feedback resulted in a lengthening of the perturbed press,

454 whereas a time advancement resulted in a shortening. Previous studies have primarily

455 investigated feedback delays (Furuya and Soechting 2010; Howell and Archer 1984;

456 Sakata and Brainard 2006; van der Steen et al. 2014) but have rarely advanced

457 participants' feedback (Repp 2002; Wing 1977). By including both feedback delays and

458 advancements we provided evidence of the directional nature of sensory feedback

459 integration in fast finger movements. 
The reaction to the delay of haptic feedback was very fast and occurred within

$461 \quad 60-90 \mathrm{~ms}$ after the expected time of the feedback. This finding is consistent with

462 previous reports that demonstrate responses between 65-110 ms following a haptic

463 input (Abbs et al. 1984; Pruszynski et al. 2016; Scott 2016). In contrast, auditory and

464 visual feedback alone did not elicit a strong reaction on the press, consistent with the

465 fact that the fasted reactions to changes in these two modalities are noticeably slower

466 (Burnett et al. 1998; Day and Lyon 2000; Howell 2004; MacKenzie and Marteniuk 1985;

467 Smith and Bowen 1980; Veerman et al. 2008). Therefore, by including a haptic

468 feedback condition we were able to show the very rapid integration of sensory feedback

469 in the execution of a finger press.

Shift from feedback to feed-forward control with learning

While the feedback perturbation still impacted the execution of the perturbed

472 press on the last day of practice, we did find that the effect reduced by approximately

$47340 \%$ with training. This observation is in line with previous research that observed a shift

474 from feedback to feed-forward control with training (Pew 1966; Seidler-Dobrin and

475 Stelmach 1998). It has been suggested that feedback plays an important role in the

476 initial phases of acquiring a novel motor skill, but its importance decreases, and

477 potentially even disappears altogether, with prolonged training (Pew 1966; Pratt et al.

478 1994; Schmidt 1975; Schmidt and McCabe 1976; Seidler-Dobrin and Stelmach 1998).

479 The main theoretical idea here is that, as we acquire an accurate internal representation

480 of the instructed movements, sensory feedback becomes less necessary for execution

481 (MacNeilage and MacNeilage 1973; Schmidt 1975; Seidler-Dobrin and Stelmach 1998).

482 Alternatively, participants potentially learned that the large deviations of the sensory 
483 feedback were irrelevant for overall performance and therefore could be ignored (Wei

484 and Körding 2009).

485 Distinct feedback processes govern the control of rapid finger movement sequences

Our second goal was to understand how sensory feedback is being taken into

487 account in the control of a complex motor sequence. Models of sequence

488 representation fall between two opposing extremes: A single, integrated motor program,

489 and a hierarchical organization (Fig. 1). By examining how feedback is integrated

490 across multiple finger presses, we were able to investigate the underlying organizational

491 structure and how feedback is integrated across the different layers.

492 We found that the feedback perturbation on a single press also affected the

493 execution of subsequent presses, both at the beginning and at the end of training.

494 Important, the reaction to the feedback perturbation was different for the perturbed and

495 subsequent presses. This finding argues against the idea that after prolonged training a

496 movement sequence is represented as a single motor program (Keele 1968; Rozanov

497 et al. 2010), in which each finger is affected in the same way by the perturbation.

498 Instead, our results more closely align with the idea of a hierarchical organization

499 (Rosenbaum et al. 1983), in which the sequence is controlled through the interaction of

500 different layers that control sequence execution. One possible organization is a two-

501 tiered structure (Fig. 1b), in which a sequence controller is positioned at the highest

502 level representing the specific order of movements and commanding the next layer of

503 finger controllers, which in turn are responsible for the control of specific finger

504 movements. Our results suggest three characteristics of this proposed two-tiered control 505 structure: 
First, sensory feedback from the finger itself is continuously relayed to the finger controller which then impacts the ongoing movement execution in a directional specific manner. Second, upon receiving the sensory feedback signifying press completion, the

509 finger controller issues a completion signal to the sequence controller. Our finding that

510 feedback not only impacts the ongoing press but also subsequent presses, suggests

511 that information is relayed across all hierarchical levels. Third, we found that both

512 feedback advancements and delays led to an overall slower initiation of the next finger

513 in the sequence. This slowing possibly reflects a cautionary measure: The sequence

514 controller may compare a prediction of when a completion signal is expected versus

515 when it is received and, upon detecting a mismatch, delays the execution of the next

516 press to ensure the successful completion of the sequence. We also found that only the

517 two larger sensory feedback perturbations led to a significant delay, suggesting that the

518 cautionary response is proportional to the amount mismatch between expected and

519 received feedback from the lower-level controller. Additionally, the sequence controller

520 also showed a reaction to a delay or time advancement in auditory and visual feedback,

521 which did not influence the local press, indicating that the sequence controller also has

522 direct access to sensory feedback signalling whether the goal of an action has been

523 achieved.

524 Previous research studying time delays and advancements of an external pacing 525 signal in a synchronization paradigm (Furuya and Soechting 2010; Repp 2000; Wing

526 1977) also have shown evidence for feedback adjustments in a hierarchical sequence

527 controller. In contrast to our experiment, in which a feedback perturbation led to a delay 528 irrespective of direction of the perturbation, these adjustments were targeted at bringing 
529 the finger presses back into synchronization with the metronome (Furuya and Soechting

530 2010; Repp 2000). In our paradigm, the timing was not constraint by a metronome, such

531 that the task goal was not to preserve a rhythm. The difference in findings between

532 these two paradigms make it likely that the reaction of the sequence controller to

533 feedback perturbation will strongly depend on the task goal.

\section{Conclusion}

535 In this study, we demonstrated that sensory feedback is continuously used to

536 adjust movement execution, but that the extent of this integration diminishes with

537 training. Haptic feedback drove the effects we observed on the perturbed press,

538 whereas the effects across the remaining movements in the sequence were impacted

539 by the perturbation in all three feedback modalities. Lastly, we demonstrated distinct

540 types of feedback processes involved in the hierarchical control of skilled finger

\section{1 sequences.}




\section{References}

Abbs JH, Gracco VL, Cole KJ. Control of Multimovement Coordination. J Mot Behav 16: 195-232, 1984.

Adams JA. A closed-loop theory of motor learning. J Mot Behav 3: 111-150, 1971.

Aschersleben G. Temporal control of movements in sensorimotor synchronization.

551 Brain Cogn 48: 66-79, 2002.

552 Burnett TA, Freedland MB, Larson CR, Hain TC. Voice F0 responses to

553 manipulations in pitch feedback. J Acoust Soc Am 103: 3153-3161, 1998.

554 Cluff T, Crevecoeur F, Scott SH. A perspective on multisensory integration and rapid

555 perturbation responses. Vision Res 110: 215-222, 2015.

556 Cole KJ, Abbs JH. Grip force adjustments evoked by load force perturbations of a

557 grasped object. J Neurophysiol 60: 1513-1522, 1988.

558 Day BL, Lyon IN. Voluntary modification of automatic arm movements evoked by

559 motion of a visual target. Exp Brain Res 130: 159-168, 2000.

560 Diedrichsen J, Kornysheva K. Motor skill learning between selection and execution.

561 Trends Cogn Sci 19: 227-233, 2015.

562 Furuya S, Soechting JF. Role of auditory feedback in the control of successive

563 keystrokes during piano playing. Exp Brain Res 204: 223-237, 2010.

564 Gates A, Bradshaw JL, Nettleton NC. Effect of different delayed auditory feedback

565 intervals on a music performance task. Percept Psychophys 15: 21-25, 1974.

566 Hernandez-Castillo CR, Maeda RS, Pruszynski JA, Diedrichsen J. Sensory

567 information from a slipping object elicits a rapid and automatic shoulder response. J 
Neurophysiol 123: 1103-1112, 2020.

569 Howell P. Effects of delayed auditory feedback and frequency-shifted feedback on

570 speech control and some potentials for future development of prosthetic aids for

571 stammering. Stammering Res 1: 31-46, 2004.

572 Howell P, Archer A. Susceptibility to the effects of delayed auditory feedback. Percept

573 Psychophys 36: 296-302, 1984.

574 Jay C, Hubbold R. Delayed Visual and Haptic Feedback in a Reciprocal Tapping Task.

575 In: First Joint Eurohaptics Conference and Symposium on Haptic Interfaces for Virtual

576 Environment and Teleoperator Systems. IEEE, p. 655-656.

577 Johansson RS, Häger C, Bäckström L. Somatosensory control of precision grip

578 during unpredictable pulling loads. Exp Brain Res 89: 204-213, 1992.

579 Keele SW. Movement control in skilled motor performance. Psychol Bull 70: 387-403,

5801968.

581 Kiebel SJ, von Kriegstein K, Daunizeau J, Friston KJ. Recognizing Sequences of

582 Sequences. PLoS Comput Biol 5: e1000464, 2009.

583 Kulpa JD, Pfordresher PQ. Effects of delayed auditory and visual feedback on

584 sequence production. Exp Brain Res 224: 69-77, 2013.

585 Long J. Effects of Randomly Delayed Visual and Auditory Feedback on Keying

586 Performance. Ergonomics 18: 337-347, 1975.

587 MacKenzie CL, Marteniuk RG. Motor skill: Feedback, knowledge, and structural

588 issues. Can J Psychol Can Psychol 39: 313-337, 1985.

589 MacNeilage PF, MacNeilage LA. Central processes controlling speech production

590 during sleep and waking. Psychophysiol thinking Acad Press 417-448, 1973. 
591 Pew RW. Acquisition of hierarchical control over the temporal organization of a skill. J

592 Exp Psychol 71: 764-771, 1966.

593 Pfordresher PQ, Benitez B. Temporal coordination between actions and sound during

594 sequence production. Hum Mov Sci 26: 742-756, 2007.

595 Pratt J, Chasteen AL, Abrams RA. Rapid aimed limb movements: Age differences and

596 practice effects in component submovements. Psychol Aging 9: 325-334, 1994.

597 Pruszynski JA, Johansson RS, Flanagan JR. A Rapid Tactile-Motor Reflex

598 Automatically Guides Reaching toward Handheld Objects. Curr Biol 26: 788-792, 2016.

599 Repp BH. Compensation for subliminal timing perturbations in perceptual-motor

600 synchronization. Psychol Res Psychol Forsch 63: 106-128, 2000.

601 Repp BH. Automaticity and voluntary control of phase correction following event onset

602 shifts in sensorimotor synchronization. J Exp Psychol Hum Percept Perform 28: 410_

$603430,2002$.

604 Rosenbaum DA, Kenny SB, Derr MA. Hierarchical control of rapid movement

605 sequences. J Exp Psychol Hum Percept Perform 9: 86-102, 1983.

606 Rozanov S, Keren O, Karni A. The specificity of memory for a highly trained finger

607 movement sequence: Change the ending, change all. Brain Res 1331: 80-87, 2010.

608 Sakata JT, Brainard MS. Real-time contributions of auditory feedback to avian vocal

609 motor control. J Neurosci 26: 9619-9628, 2006.

610 Schmidt RA. A schema theory of discrete motor skill learning. Psychol Rev 82: 225-

$611260,1975$.

612 Schmidt RA, McCabe JF. Motor program utilization over extended practice. J Hum

613 Mov Stud 2: 239-247, 1976. 
614 Scott SH. The computational and neural basis of voluntary motor control and planning.

615 Trends Cogn Sci 16: 541-549, 2012.

616 Scott SH. A Functional Taxonomy of Bottom-Up Sensory Feedback Processing for

617 Motor Actions. Trends Neurosci 39: 512-526, 2016.

618 Seidler-Dobrin RD, Stelmach GE. Persistence in visual feedback control by the

619 elderly. Exp Brain Res 119: 467-474, 1998.

620 Shadmehr R, Smith MA, Krakauer JW. Error correction, sensory prediction, and

621 adaptation in motor control. Annu Rev Neurosci 33: 89-108, 2010.

622 Smith WM, Bowen KF. The Effects of Delayed and Displaced Visual Feedback on

623 Motor Control. J Mot Behav 12: 91-101, 1980.

624 van der Steen MC, Molendijk EBD, Altenmüller E, Furuya S. Expert pianists do not

625 listen: The expertise-dependent influence of temporal perturbation on the production of

626 sequential movements. Neuroscience 269: 290-298, 2014.

627 Veerman MM, Brenner E, Smeets JBJ. The latency for correcting a movement

628 depends on the visual attribute that defines the target. Exp Brain Res 187: 219-228,

6292008.

630 Wei K, Körding K. Relevance of error: What drives motor adaptation? J Neurophysiol

631 101: 655-664, 2009.

632 Wiestler T, Diedrichsen J. Skill learning strengthens cortical representations of motor

633 sequences. Elife 2: 1-20, 2013.

634 Wiestler T, Waters-Metenier S, Diedrichsen J. Effector-Independent Motor Sequence

635 Representations Exist in Extrinsic and Intrinsic Reference Frames. J Neurosci 34:

636 5054-5064, 2014. 
bioRxiv preprint doi: https://doi.org/10.1101/2021.07.02 450916; this version posted July 3, 2021. The copyright holder for this preprint (which was not certified by peer review) is the author/funder, who has granted bioRxiv a license to display the preprint in perpetuity. It is made available under aCC-BY-NC-ND 4.0 International license.

637 Wing AM. Perturbations of auditory feedback delay and the timing of movement. J Exp

638 Psychol Hum Percept Perform 3: 175-186, 1977.

639 Yokoi A, Bai W, Diedrichsen J. Restricted transfer of learning between unimanual and

640 bimanual finger sequences. J Neurophysiol 117: 1043-1051, 2017.

641 